\title{
A utilidade do Miniexercício Clínico Avaliativo (Miniex) como ferramenta padrão na graduação de fisioterapia
}

\author{
The usefulness of the Evaluative Clinical Mini Exercise (Miniex) as a standard tool in \\ physiotherapy graduation
}

\section{La utilidad del Mini Ejercicio Clínico Evaluativo (Miniex) como herramienta estándar en la graduación de fisioterapia}

Luiz Euclides Coelho de Souza Filho ${ }^{1 *}$, Katiane da Costa Cunha ${ }^{2}$, Marcio Clementino de Souza Santos $^{1}$, Luiz Fábio Magno Falcão ${ }^{1}$, Valéria Marques Ferreira Normando.

\section{RESUMO}

Objetivo: Avaliar o uso do Miniexercício Clínico Avaliativo (Miniex) como ferramenta útil no cenário de prática sob a visão discente. Métodos: Estudo do tipo single-arm clinical trail, descritivo e comparativo do tipo antes e depois com discentes $(n=30)$ de graduação do último ano de Fisioterapia de uma Universidade Pública no Estado do Pará, no período de agosto a dezembro de 2018, sendo utilizado o questionário traduzido Miniex. Resultados: $96 \%$ dos discentes alegam o padrão avaliativo do Miniex ser mais objetivo do que o tradicional; $92 \%$ dos discentes consideram que o Miniex deveria ser o padrão de exame no currículo de fisioterapia; $52 \%$ aceitaram que esta ferramenta avalia o discente completamente; $88 \%$ dos discentes aceitaram que o tempo atribuído era satisfatório, enquanto $40 \%$ dos discentes ficaram desconfortáveis, enquanto avaliavam o paciente na presença do professor; $92 \%$ dos discentes concordaram que, em geral, é uma maneira muito eficaz de avaliação do discente de Fisioterapia e $84 \%$ concordam que podem obter melhor pontuação em exames futuros. Conclusão: $O$ instrumento Miniex revelou poder ser uma ferramenta útil no cenário de prática real à fisioterapia contribuindo para a construção e o desenvolvimento desses futuros profissionais.

Palavras-chave: Fisioterapia, Avaliação educacional, Unidade de terapia intensiva.

\begin{abstract}
Objective: To evaluate the use of the Clinical Mini-Exercise (Mini-Exercise) as a useful tool in the practice scenario under the student's view. Methods: Study of the single-arm clinical trail type, descriptive and comparative of the type before and after with undergraduate students $(n=30)$ from the last year of Physiotherapy at a Public University in the State of Pará, from August to December 2018, using the translated questionnaire Miniex. Results: $96 \%$ of the students claim that the evaluation standard of Miniex is more objective than the traditional one; $92 \%$ of the students consider that the Miniex should be the standard of examination in the physiotherapy curriculum; $52 \%$ accepted that this tool evaluates the student completely; $88 \%$ of the students accepted that the time allotted was satisfactory, while $40 \%$ of the students were uncomfortable, while evaluating the patient in the presence of the teacher; $92 \%$ of the students agreed that, in general, it is a very effective way of evaluating the Physiotherapy student and $84 \%$ agree that they can obtain a better score in future exams. Conclusion: The Miniex instrument proved to be a useful tool in the real practice of physical therapy, contributing to the construction and development of these future professionals.
\end{abstract}

Keywords: Physiotherapy, Educational measurement, Intensive care units.

\section{RESUMEN}

Objetivo: Evaluar el uso del Mini-Ejercicio Clínico (Mini-Ejercicio) como una herramienta útil en el escenario de práctica bajo la mirada del alumno. Métodos: Estudio del tipo de recorrido clínico monobrazo, descriptivo y comparativo del tipo antes y después con estudiantes de pregrado $(n=30)$ del último año de Fisioterapia en una Universidad Pública del Estado de Pará, de agosto a diciembre de 2018. , utilizando el cuestionario traducido Miniex. Resultados: El 96\% de los estudiantes afirma que el estándar de evaluación del Miniex es más objetivo que el tradicional; El 92\% de los estudiantes consideró que el Miniex debería ser el estándar de examen en el plan de estudios de fisioterapia; El 52\% aceptó que esta herramienta evalúa al alumno en su totalidad; El $88 \%$ de los estudiantes aceptó que el tiempo asignado fue satisfactorio, mientras que el $40 \%$ de

1 Universidade do Estado do Pará (UEPA), Belém - PA. *E-mail: luizcoelhodesouza@yahoo.com.br

2 Universidade Federal do Pará (UFPA), Belém - PA. 
los estudiantes se mostró incómodo al evaluar al paciente en presencia del profesor; El 92\% de los estudiantes coincidió en que, en general, es una forma muy eficaz de evaluar al estudiante de Fisioterapia y el $84 \%$ está de acuerdo en que puede obtener una mejor puntuación en futuros exámenes. Conclusión: El instrumento Miniex demostró ser una herramienta útil en la práctica real de la fisioterapia, contribuyendo a la construcción y desarrollo de estos futuros profesionales.

Palabra clave: Fisioterapia, Evaluación educacional, Unidades de cuidados intensivos.

\section{INTRODUÇÃO}

No contexto educacional, avaliar significa emitir juízo sobre a aprendizagem adquirida, planejada e sistematizada visando a evolução educacional do ser humano. Quando somativa, informa ao avaliador sobre a classificação por meio de notas ou conceitos, já a formativa possui o foco na aprendizagem. Há a identificação dos pontos fortes e fracos do avaliado tendo o feedback como a matriz principal dessa avaliação. Apesar dessa dicotomia teórica, ambas podem viver em comunhão e se alternarem no processo avaliativo, fato que pode ocorrer no ensino das profissões da saúde (PANÚNCIO-PINTO MP e TRONCON LE, 2014).

A escolha do método avaliativo empregado é de fundamental importância sendo o modelo conceitual da Pirâmide de Miller muito utilizado nas decisões. Formado por quatro partes onde a base da pirâmide é o "saber", seguido do "saber como fazer", "mostrar como faz" e, por fim, o "fazer". O estrato do "fazer" está intimamente ligado ao local onde a prática é exercida, ou seja, ao ambiente de trabalho (PANÚNCIO-PINTO MP e TRONCON LE, 2014).

Na graduação em saúde o estágio pode ser visto como um período de prática para que uma determinada pessoa se habilite em algo. Essa habilitação pode estar vinculada a fatos diversos e, também, a profissão escolhida. Está ligada a treino, exercício, atividade prática e pode ser considerado obrigatório quando da formação de um discente em busca de uma graduação profissional. Independente dos muitos conceitos existentes suas definições sempre estão pautadas em atividades práticas. Mostra-se como um trabalho temporário em que se insere um contrato temporário entre os autores do processo (instituições, empresas e órgãos) (BRASIL, 2002; ARAUJO FRO, et al., 2010).

No processo de formação acadêmica na área da saúde, o conhecimento prático é tão importante quanto o teórico, sendo o saber fazer ponto fundamental avaliado nos campos de estágio e uma das bases de formação deste futuro profissional. Para tal, o estágio na saúde é espaço pedagógico fundamental para a formação deste futuro profissional e elo do conhecimento teórico-prático. O estágio supervisionado (ES) promove a conjunção da formação teórico-científica com as situações do cotidiano essenciais na formação do profissional de saúde (MOTTER AA, et al., 2014).

Diante do "fazer", o Mini-Clinical Exercise (Mini-CEX), criado e elaborado para avaliação na prática da medicina consiste em, a partir da condução de um plano diagnóstico e terapêutico pelo residente, que ocorra a avaliação do docente com feedback educacional (NORCINI JJ, et al., 1995; MEGALE L, et al., 2009; PANÚNCIO-PINTO MP e TRONCON LE, 2014).

O referido instrumento foi relatado na literatura em 1995, por meio do estudo de Norcini et al. (1995) intitulado "The Mini-CEX (Clinical Evaluation Exercise): A Preliminary Investigation". Habilidades na entrevista médica, no exame físico e de orientação, assim como qualidades humanísticas/profissionalismo, raciocínio, organização/eficiência são os tópicos avaliados pela ferramenta. Sua tradução e validação para a Língua Portuguesa modificou seu nome de Mini-CEX para Miniex (MEGALE L, et al., 2009).

Como avaliação do desempenho em situação real, problemas são enfrentados como a falta de padronização, observações limitadas, viés e amostragem limitada de habilidades clínicas, o que limita o uso em exames de qualificação. Apesar de ser muito utilizado com os discentes de medicina, o presente instrumento ainda não foi estudado com ênfase para outros graduandos da saúde, tendo no âmbito da Fisioterapia uma lacuna avaliativa para cenários de prática reais (RATHI M e GAZBARE P, 2017).

Diante da falta de ferramentas de avaliação para o cenário de prática real no âmbito da graduação em Fisioterapia o presente estudo visa avaliar o uso do Miniexercício Clínico Avaliativo (Miniex) como uma ferramenta útil no cenário de prática sob a visão discente. 


\section{MÉTODOS}

Estudo do tipo single-arm clinical trail, descritivo e comparativo do tipo antes e depois, foi realizado no período de agosto a dezembro de 2018, após aprovação no Comitê de Ética e Pesquisa com Seres Humanos de um Hospital Estadual Referência em Oncologia (Parecer no 2.395.967), local onde foi realizado o estudo, mais especificamente na Unidade de Terapia Intensiva adulto (UTI).

Trinta discentes do $8^{\circ}$. semestre regularmente matriculados no Curso de Fisioterapia de uma Universidade Pública do Estado do Pará, de ambos os sexos, fizeram parte do estudo após assinatura do Termo de Consentimento Livre e Esclarecido (TCLE).

Foram excluídos os que estivessem em situação de dependência ou outra situação que não fosse o primeiro contato com a disciplina, discentes que estivessem realizando ou tivessem realizado estágio extracurricular na área de terapia intensiva ou qualquer situação que pudesse interferir na avaliação prática dos discentes (Figura 1).

Aptos à avaliação dos discentes estiveram os pacientes com idade maior de 18 anos, em uso de assistência ventilatória invasiva e ausência de restrição terapêutica curativa. Conduziu-se o registro numérico pelo pesquisador e sorteados pelos discentes participantes. Todos os responsáveis pelos pacientes autorizaram a coleta de dados a partir da assinatura do Termo de Assentimento.

Como instrumento protocolar foi utilizado o Miniex, traduzido e validado para o Português (MEGALE L, et al., 2009), sendo conduzidos por avaliações entre 15 e 20 minutos, com uma escala numérica de 9 pontos, sendo considerado insatisfatório de 1 a 3 , satisfatório de 4 a 6 e superior de 7 a 9 . Os quesitos avaliados pelo examinador foram as habilidades na entrevista, exame físico, qualidades humanísticas/profissionalismo, raciocínio clínico, habilidades de orientação, organização/eficiência e competência clínica geral. Vale ressaltar que o pesquisador entrou em contato com o Dr. John Norcini, idealizador do instrumento, para solicitar autorização no uso do instrumento para o ambiente prático fisioterapêutico.

Ao término da segunda avaliação foi utilizado um questionário, usado na pesquisa de Rathi $\mathrm{M}$ e Gazbare P (2017), para avaliar o grau de satisfação dos discentes com o uso da ferramenta Miniex de avaliação tendo em vista a pouco utilização no âmbito da prática discente fisioterapêutica. Este foi traduzido do Inglês para o Português pelos autores da pesquisa.

Figura 1 - Seleção dos 30 discentes aptos a responder ao questionário Miniex.

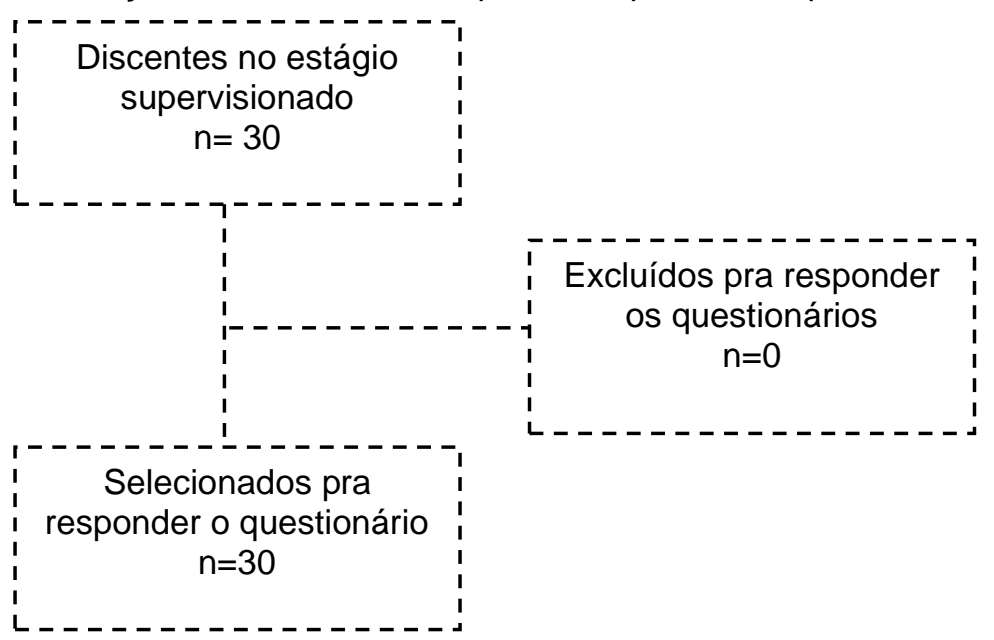

Fonte: Filho LECS, et al., 2021.

Os trinta discentes foram avaliados quanto à sua prática de avaliação do paciente crítico no primeiro (T1) e no último dia do estágio (T9). Essa avaliação foi realizada por um avaliador independente, que foi instruído pelo pesquisador sobre o Miniex. Estes transitaram em grupos de cinco, em dias intercalados, por três semanas ininterruptas na Unidade de Terapia Intensiva do HOL. 
Entre a primeira e a última avaliação realizadas pelos avaliadores convidados, os trinta discentes tiveram sua prática supervisionada na UTI normalmente, de acordo com o cronograma da Instituição a qual pertenciam.

A pesquisa ocorreu em sete etapas: (etapa 1) seleção pelo pesquisador dos pacientes a serem avaliados; (etapa 2) sorteio dos pacientes realizado por cada um dos discentes; (etapa 3) entrega da folha do Miniex ao avaliador independente no primeiro dia de estágio supervisionado; (etapa 4) realização da atividade prática elaborada pela Instituição de ensino e supervisionada pelo preceptor responsável, (etapa 5) pesquisador seleciona os pacientes a serem avaliados, (etapa 6) sorteio dos pacientes realizado por cada um dos discentes e (etapa 7) entrega de nova folha do Miniex no último dia de estágio supervisionado ao avaliador independente e entrega do questionário avaliativo do instrumento de avaliação aos discentes.

Inicialmente, na etapa 1, o pesquisador selecionou os pacientes a serem avaliados pelos discentes, numerando-os de acordo com o leito ocupado pelo paciente. Vale enfatizar que os pacientes foram avaliados anteriormente na ausência dos discentes pelo avaliador independente, pesquisador e pelo fisioterapeuta do setor. Em seguida, na etapa 2, os discentes sortearam o paciente que cada um deveria avaliar. Na etapa 3 , o avaliador independente recebeu a folha do Miniex e encaminhou os discentes até o leito do paciente sorteado por eles. Vale ressaltar que o Miniex foi utilizado pelo examinador independente e foi solicitado ao avaliador que nesta primeira avaliação não fosse dado feedback aos discentes.

Posteriormente, na etapa 4, os discentes participavam de suas atividades normais no cenário de prática real, UTI, seguindo as regras da Instituição Educacional.

$\mathrm{Na}$ etapa 5, ocorreu uma nova seleção, por parte do pesquisador, de pacientes a serem avaliados pelos discentes, assim como ocorrera na etapa 1. Vale enfatizar que os pacientes eram avaliados antes pelo avaliador independente, pesquisador e pelo fisioterapeuta do setor.

$\mathrm{Na}$ etapa 6, os discentes realizaram um novo sorteio e eram novamente encaminhados para avaliar seus respectivos pacientes, como descrito na etapa 2. Por fim, na etapa 7, novamente 0 avaliador independente reavaliou os discentes por meio do questionário Miniex, sendo desta vez dado feedback aos discentes, como a ferramenta exige. Além disso foi dado o questionário avaliativo da ferramenta aos discentes.

A busca por artigos se deu nas bases de dados Medline, Pubmed, Eric, Bireme e Google acadêmico. Foram usados os descritores do DESC/MESH, bem como o thesaurus no Eric. Os descritores utilizados foram os seguintes: "Unidade de Cuidados Intensivos", "Discentes", "student", "ungraduate", "university", "academic", "icu", "intensive care unit", "intensive care units", "physiotherapist", "physiotherapy". Foram utilizadas as palavras chaves "minicex", "mini cex", "Miniex", "clinical evoluation exercise".

As informações da caracterização amostral, após coleta, foram compiladas em banco de dados elaborado no software Microsoft $\circledast$ Office Excel® 2016.

\section{RESULTADOS}

Os achados da presente pesquisa foram organizados em: características dos discentes, desempenho dos discentes na avaliação do paciente crítico e avaliação da ferramenta Miniex no cenário de prática. Abaixo vimos a divisão por faixa etária e gênero dos participantes da pesquisa não sendo demonstrado diferença entre as proporções de faixa etária com amostra homogênea (Tabela 1).

Tabela 1 - Faixa etária em anos dos discentes de Fisioterapia.

\begin{tabular}{ccc}
\hline Faixa etária (em anos) & Frequência & $\%$ \\
\hline 20 a 21 & 15 & $50,0 \%$ \\
22 a 23 & 06 & $20,0 \%$ \\
24 a 25 & 06 & $20,0 \%$ \\
> 25 & 03 & $10,0 \%$ \\
Total & 30 & $100,0 \%$ \\
\hline
\end{tabular}

Legenda: $p=0,0085$ Teste $G$ Aderência.

Fonte: Filho LECS, et al., 2018. 
Tabela 2 - Frequência por gênero dos discentes de Fisioterapia.

\begin{tabular}{ccc}
\hline SEXO & Frequência & $\%$ \\
\hline Feminino* $^{*}$ & 23 & $77,0 \%$ \\
Masculino $_{\text {Total }}$ & 7 & $23,0 \%$ \\
& 30 & $100,0 \%$ \\
\hline
\end{tabular}

Legenda: ${ }^{*} p=0,0062$ Teste Qui-Quadrado Aderência.

Fonte: Filho LECS, et al., 2021.

A tabela 3 mostra a resposta em percentual dos 30 participantes quanto ao questionário sobre a utilização do Miniex como ferramenta de avaliação no cenário de prática (Tabela 3).

Tabela 3 - Utilização do Miniex como ferramenta de avaliação no cenário de prática.

\begin{tabular}{|c|c|c|c|}
\hline QUESTIONÁRIO AVALIATIVO DO Mini-Ex & Concordância & Discordância & Neutro \\
\hline \multicolumn{4}{|l|}{ Afirmativas Positivas } \\
\hline $\begin{array}{l}\text { Q } 01 \text { - O padrão avaliativo do Miniex é mais objetivo } \\
\text { que o tradicional. }\end{array}$ & $96,0 \%$ & $4,0 \%$ & $0,0 \%$ \\
\hline $\begin{array}{l}\text { Q } 02 \text { - Avalia completamente nosso conhecimento, } \\
\text { habilidade e atitude. }\end{array}$ & $52,0 \%$ & $24,0 \%$ & $24,0 \%$ \\
\hline $\begin{array}{l}\text { Q } 03 \text { - Melhora o nosso desempenho no exame da } \\
\text { Universidade, pois o feedback foi oportuno. }\end{array}$ & $92,0 \%$ & $0,0 \%$ & $8,0 \%$ \\
\hline Q 04 - O tempo atribuído à avaliação foi apropriado. & $88,0 \%$ & $8,0 \%$ & $4,0 \%$ \\
\hline $\begin{array}{l}\text { Q } 05 \text { - Esta avaliação deve ser incluída no currículo } \\
\text { do exame prático. }\end{array}$ & $92,0 \%$ & $4,0 \%$ & $4,0 \%$ \\
\hline $\begin{array}{l}\text { Q } 06 \text { - É bom aprender a diagnosticar habilidades em } \\
\text { curto período de tempo. }\end{array}$ & $88,0 \%$ & $0,0 \%$ & $12,0 \%$ \\
\hline $\begin{array}{l}\text { Q } 08 \text { - Sentiu-se satisfeito com o padrão de } \\
\text { avaliação, pois o professor me observou enquanto } \\
\text { avaliava. }\end{array}$ & $76,0 \%$ & $16,0 \%$ & $8,0 \%$ \\
\hline $\begin{array}{l}\text { Q } 09 \text { - Este tipo de avaliação nos ajudará a examinar } \\
\text { adequadamente o paciente. }\end{array}$ & $88,0 \%$ & $4,0 \%$ & $8,0 \%$ \\
\hline $\begin{array}{l}\text { Q } 11 \text { - Podem obter melhor pontuação com esta } \\
\text { forma de avaliação. }\end{array}$ & $84,0 \%$ & $4,0 \%$ & $12,0 \%$ \\
\hline $\begin{array}{l}\text { Q } 12 \text { - No geral, é uma maneira muito eficaz de } \\
\text { avaliação. }\end{array}$ & $92,0 \%$ & $0,0 \%$ & $8,0 \%$ \\
\hline
\end{tabular}

\begin{tabular}{lccc}
\hline Afirmativas Negativas & & & \\
\hline $\begin{array}{l}\text { Q } 07 \text { - Torna-se difícil fazer o diagnóstico neste curto } \\
\text { espaço de tempo. }\end{array}$ & $20,0 \%$ & $60,0 \%$ & $20,0 \%$ \\
$\begin{array}{l}\text { Q } 10 \text { - Sentiu-se desconfortável ao avaliar o paciente } \\
\text { na frente do professor. }\end{array}$ & $40,0 \%$ & $28,0 \%$ & $32,0 \%$
\end{tabular}

Fonte: Filho LECS, et al., 2021.

\section{DISCUSSÃO}

Um dos pontos mais importantes no processo de ensino aprendizagem e a avaliação discente, pois por meio desta o docente e o discente conseguem ver a evolução educacional e prática, podendo intervir para lapidar esse discente em busca do objetivo que almeja. Dentro do quesito avaliativo, há diversas ferramentas para avaliações teóricas e práticas. Para a avaliação prática, foi escolhida a ferramenta de avaliação Miniex 
por ser um instrumento que avalia o discente o mais próximo do cenário de prática que ele encontrará na vida profissional. Essa ferramenta avalia as habilidades e competências correspondendo ao estrato do "mostrar como faz" da Pirâmide de Miller (PANÚNCIO-PINTO MP e TRONCON LE, 2014).

Diversos autores da área fizeram resvisões sistemáticas sobre ferramentas de avaliação, em que foi verificado escassez destas para os cenários de prática Fisioterapêutica (DA SILVA AM, et al., 2014 e HENGSOMBOON N, 2017 e O'CONNOR A, et al., 2018). Tal observação mostra a lacuna em achar e utilizar instrumentos adequados de avaliação discente nos cenários de prática tendo que ser utilizado instrumentos criados e validados para outras áreas da saúde. Ademais, até o presente momento não há na literatura ferramentas de avaliação para o cenário de prática na nossa língua mãe o que dificulta a inserção das ferramentas já existentes no dia a dia da prática supervisionada (O'CONNOR A, et al., 2018).

O questionário passado aos discentes mostra um alto grau de satisfação quanto à ferramenta utilizada para avaliação na pesquisa. Esse resultado corrobora com a pontuação encontrada no estudo de tradução e validação do instrumento em que foi verificado um índice de satisfação de 8,3 pontos em uma escala de até 9 pontos por parte dos discentes (MEGALE L, et al., 2009). Além deste, o estudo das pesquisadoras Rathi M e Gazbare P (2017) monstraram pontuações positivas similares ao presente estudo e ao estudo supracitado mostrando a ferramenta avaliativa como uma boa opção para cenários de prática real.

Para tal, houve a adesão de 30 discentes com homogeneidade frente a faixa etária e heterogeneidade quando ao gênero, havendo uma predominância do sexo feminino $(p<0.05)$. A heterogeneidade quando ao gênero é similar aos estudos de Badaró AFV e Guilhem D (2011) e Santos WV, et al. (2017) que verificaram uma população feminina maior em relação à masculina (Tabela 2).

A Tabela 3 mostra o feedback dos discentes sobre o Miniex. No presente estudo, $96 \%$ dos discentes aceitaram que o Miniex é o padrão mais objetivo do que o tradicional. $92 \%$ dos discentes consideram que 0 Miniex deveria ser o padrão de exame no currículo de fisioterapia; $52 \%$ aceitaram que esta ferramenta avalia o discente completamente. $88 \%$ dos discentes aceitaram que o tempo atribuído era satisfatório enquanto $40 \%$ dos discentes ficaram desconfortáveis, enquanto avaliavam o paciente na presença do professor. $92 \%$ dos discentes concordaram que, em geral, é uma maneira muito eficaz de avaliação do discente de Fisioterapia e $84 \%$ concordam que podem obter melhor pontuação em exames futuros.

No estudo de Rathi M e Gazbare P (2017), os valores encontrados foram de $100 \%, 80 \%, 90 \%, 75 \%, 35 \%$, $85 \%$ e $100 \%$, respectivamente. Tal estudo mostra números bem similares com exceção do quesito 02 "avalia completamente nosso conhecimento, habilidade e atitude" em que há uma diferença do estudo atual (52\%) para o estudo analisado (90\%).

Estudo de Suhoyo Y, et al. (2018), mostrou que, em geral, estudantes e especialistas foram positivos a muito positivos quanto à praticidade do Miniex e ao impacto desse formato de avaliação na aprendizagem e no desenvolvimento profissional. Essas características verificadas nos presentes estudos podem justificar o grande uso do Mini-CEX nos últimos anos em diversas áreas acadêmicas da saúde, bem como um aumento no estudo dessa ferramenta por essas graduações da saúde (RATHI M e GAZBARE P, 2017; JASEMI M, et al., 2019; FERNANDES BC, et al., 2020; MARTINSEN SSS, et al., 2021; VÉLIZ C et al., 2021). Vale ressaltar que a praticidade do instrumento aliado ao baixo custo são uma boa aposta para sua utilização em uma ampla gama de estágios e ambientes de trabalho (MARTINSEN SSS, et al., 2021).

O presente estudo observou que o instrumento Miniex aparenta ser mais objetivo do que o método de avaliação tradicional, fato analisado pela percepção dos discentes entrevistados, em que foi verificado uma concordância de 96\% para a questão 1 (HILL F, et al., 2009; LIAO KC, et al., 2013; ALVES DE LIMA A, et al., 2013 e RATHI M e GAZBARE P, 2017). Assim como no presente estudo, Rathi M e Gazbare P (2017) confirmam com os achados de que o Miniex pode ser o padrão de exame no currículo da Fisioterapia. Já pode-se ler estudos usando a ferramenta Mini-CEX na prática da Fisioterapia a nível nacional, porém os estudos ainda são iniciais e em pequena quantidade quando comparado com outras profissões. Apesar disto, os poucos estudos já demonstram resultados positivos que podem e devem ser levandos em consideração para estudos futuros (SIQUEIRA JC, 2018). 
Segundo o estudo de Hejri SM, et al. (2017), o mini-CEX é amplamente utilizado como uma ferramenta de avaliação formativa e somativa e parece ter validade e confiabilidade razoáveis. A aceitabilidade e viabilidade relatadas devem ser interpretadas à luz do número necessário de encontros necessários para alcançar a confiabilidade desejada. Ao fornecer uma estrutura para observação e feedback estruturados, o mini-CEX tem um impacto educacional favorável. Tais achados corroboram com os achados do presente estudo em que houve respostas positivas consideráveis nas questões 11 e 12, demonstrando tal ferramenta ser uma grande saída à evolução educacional desses futuros egressos.

Lorwald AC, et al. (2018) e Suhoyo Y, et al. (2020), revelaram um efeito positivo do Mini-CEX no desempenho do estagiário. Estes relatam que a condução adequada do Mini-CEX, incluindo uma reflexão realista do desempenho do estagiário durante a observação direta, seguida de uma conversa construtiva de feedback, parece ser um pré-requisito para seu impacto educacional. Estudo de O'Connor A, et al. (2020) demonstra que a opinião das partes interessadas no processo educacional é cada vez mais importante para o planejamento, organização e elaboração de políticas relacionadas à saúde sendo nesse contexto fundamental o feedback positivo dos discentes quando à ferramenta proposta.

Estudo de Siqueira JC (2018) que utilizou a ferramenta Mini-CEX com 10 preceptores e 14 discentes demonstrou resultados positivos por parte de ambos os grupos quanto ao feedback do uso da ferramenta. Ademais, os preceptores relataram melhora por parte dos alunos na evolução das habilidades e competências dos estudantes e estes relataram a ferramenta e seu feedback serem positivos em suas melhorias para a prática em cenários reais.

O presente estudo escolheu a ferramenta Mini-CEX para uso na pesquisa, pois esta está sendo muito utilizada no meio acadêmico da fisioterapia, porém vale ressaltar que ambas as traduções encontradas para a língua portuguesa, do Brasil e de Portugal, apesar de terem passado por todo o processo de tradução, retradução e validação foram testadas e validades no âmbito da medicina, não sendo achado tal ferramenta com o mesmo complexo processo de validação, adequação e testagem para a graduação de Fisioterapia (MEGALE L, et al., 2009; SOUSA R, et al., 2020; MARTINSEN SSS, et al., 2021).

A presente pesquisa cursou com algumas dificuldades tais como avaliar os discentes em um cenário de prática real visto ter sido a primeira vez da realização da pesquisa no cenário utilizado, evitar o vazamento de informações para as sub turmas subsequentes acerca do que seria avaliado, pois estes passavam no estágio em sub turmas pequenas e em períodos distintos, número limitado de participantes devido o quantitativo de alunos da Universidade em questão, longo tempo de coleta para o perfil do projeto que ocorreu por um semestre e utilizar uma ferramenta de avaliação não criada e disseminada no meio da Fisioterapia.

Desta forma, os autores do estudo sugerem novas pesquisas no foco em questão, com populações maiores, com adaptação da ferramenta para a graduação em questão, afim de gerar maior robustez aos resultados e cenários de prática diversos, assim como a continuidade do estudo com os discentes inseridos nas novas metodologias de ensino implementadas em várias Universidades.

\section{CONCLUSÃO}

O instrumento de avaliação Miniex revelou poder ser uma ferramenta útil no cenário de prática real à fisioterapia contribuindo para a construção e o desenvolvimento desses futuros profissionais. O feedback dado pelos alunos por meio das respostas do questionário mostram a possibilidade de um novo caminho a ser seguindo quanto à avaliação nos cenários de prática em ambiente real. Apesar desta ser uma ferramenta prática para cenários de prática real, ter passado por todo o processo de tradução, retradução e validação na Língua Portuguesa no Brasil e em Portugal, a sua construção tendo ocorrida para a educação médica, sem testagem específica na prática fisioterapêutica, mostra que sua utilização em ambientes reais da Fisioterapia ainda pode apresentar barreiras ao seu uso por completo tendo a sua utilização na prática da Fisioterapia a busca por adequações para esta outra realidade. 


\section{REFERÊNCIAS}

1. ALVES DE LIMA A, et al. A laboratory study on the reliability estimations of the mini-CEX. Advances in health sciences education: theory and practice. 1 nd Ed Springer, 2013; 18(1): 5-13.

2. ARAÚJO FRO, et al. Estágio curricular para os cursos de graduação em fisioterapia: recomendações da Abenfisio. Fisioterapia Brasil, 2010; 11: 12-15.

3. BADARÓ AFV, GUILHEM D. Perfil sociodemográfico e profissional de fisioterapeutas e origem das suas concepções sobre ética. Fisioter. mov. (Impr.), 2011; 3: 445-454.

4. BRASIL. Ministério da Educação. Conselho Nacional de Educação. Parecer CNE/CP 28/2001, de 2 de outubro de 2001. Dá nova redação ao Parecer CNE/CP 21/2001, que estabelece a duração e a carga horária dos cursos de Formação de Professores da Educação Básica, em nível superior, curso de licenciatura, de graduação plena. Diário Oficial da União: Seção 1, Brasília, DF, 2002; 31.

5. DA SILVA AM et al. Instrumentos para avaliar a prática baseada em evidências na fisioterapia: uma revisão sistemática. Com Scientia e Saúde, 2015; 14: 321-327.

6. FERNANDES BC et al. Development of MiniCEX to assess nurses' clinical skills concerning medication. Rev. Mundo da Saúde, 2020; 44: 465-485.

7. HEJRI SM et al. The utility of mini-clinical evaluation exercise (mini-CEX) in undergraduate and post graduate medical education: Protocol for a systematic review. Syst Rev, 2017; 6:1-8.

8. HENGSOMBOON N et al. Development of Physical Therapy Practical Assessment System by Using Multisource Feedback. International Education Studies, 2017; 10: 118-123.

9. HILL F et al. Implementing the undergraduate mini-CEX: a tailored approach at Southampton University. Med Educ., 2009; 43: 326-34.

10. JASEMI M et al. Are workplace-based assessment methods (DOPS and Mini-CEX) effective in nursing students' clinical skills? A single-blind randomized, parallel group, controlled trial. Contemporary nurse, 2019; 55: 565-575.

11. LIAO KC. Development and implementation of a mini-Clinical Evaluation Exercise (mini-CEX) program to assess the clinical competencies of internal medicine residents: from faculty development to curriculum evaluation. BMC Med Educ, 2013; 26: 1-7.

12. LORWALD AC. et al. The educational impact of Mini-Clinical Evaluation Exercise (Mini-CEX) and Direct Observation of Procedural Skills (DOPS) and its association with implementation: A systematic review and meta-analysis. PloS one, 2018; 13: 1-15.

13. MARTINSEN SSS et al. Examining the educational impact of the mini-CEX: a randomised controlled study. BMC medical education, 2021; 21: 1-10.

14. MEGALE L et al. Avaliação de competência clínica em estudantes de medicina pelo Miniexercício Clínico Avaliativo (Miniex). Revista Brasileira de Educação Médica, 2009; 33: 166-175.

15. MOTTER AA et al. Sentimentos vivenciados por acadêmicos de fisioterapia ao estagiar em unidade de terapia intensiva. Cad Edu Saude e Fis., 2014; 1: 73-84.

16. MORTAZ HS et al. The utility of mini-Clinical Evaluation Exercise in undergraduate and postgraduate medical education: A BEME review: BEME Guide No. 59. Medical Teacher, 2020; 42: 125-142.

17. NORCINI JJ. et al. The mini-CEX (clinical evaluation exercise): a preliminary investigation. Annals of internal medicine, 1995; 123: 795-799.

18. O'CONNOR A et al. Clinical performance assessment tools in physiotherapy practice education: a systematic review. Physiotherapy, 2018; 104: 46-53.

19. O'CONNOR A et al. Investigating physiotherapy stakeholders' preferences for the development of performance-based assessment in practice education. Physiotherapy, 2020; 108: 46-54.

20. PANÚNCIO-PINTO MP, DE ALMEIDA TRONCON LE. Avaliação do estudante-aspectos gerais. Medicina (Ribeirão Preto. Online), 2014; 47: 314-323.

21. RATHI M, GAZBARE P. Effectiveness of mini-clinical evaluation exercise (Mini CEX) in assessment of physiotherapy students. Int J Pharm Bio Sci., 2017; 8: 107-111.

22. SANTOS WV et al. Estudo do perfil e da satisfação profissional do egresso de Fisioterapia de uma instituição privada de ensino superior de 2003 a 2014. Revista Interdisciplinar Ciências Médicas - MG, 2017; 1: 16-25.

23. SIQUEIRA JC. Opinião de preceptores e estudantes de fisioterapia sobre o uso do Mini-CEX para avaliação de competências clínicas. PE. Dissertação (Mestrado em Educação em Saúde) - Faculdade Pernambucana de Saúde, Recife, 2018.

24. SOUSA R et al. Traducción, adaptación y validación del Mini-Clinical Evaluation Exercise (Mini-CEX) al idioma portugués europeo. FEM: Revista de la Fundación Educación Médica, 2020; 23: 179-192.

25. SUHOYO $Y$ et al. Clinical workplace learning: perceived learning value of individual and group feedback in a collectivistic culture. BMC medical education, 2018; 18: 1-6.

26. SUHOYO $Y$ et al. How students and specialists appreciate the mini-clinical evaluation exercise (mini- CEX) in Indonesian clerkships. BMC medical education, 2020; 20: 1-8.

27. VÉLIZ C et al. Adaptation, psychometric properties, and implementation of the Mini-CEX in dental clerkship. Journal of Dental Education, 2021; 85: 300-310. 